\title{
勤练、夯基、重梳、落规一一例谈高考物理二轮专题复习的有 效性
}

周妮娜

辽宁省鞍山市鞍钢高级中学

DOI:10.32629/er.v3i8.3103

\begin{abstract}
[摘 要] 高一高二年级进行新知识的学习,两年内完成物理考纲要求的全部内容, 进入高三后再进行 三轮复习,这是高考复习备考各高中学校常用的做法。高三的三轮复习包括一轮双基复习,二轮专题复 习, 三轮综合复习。一轮知识复习重在对教材内容进行梳理, 使同学建立高中物理知识体系, 构建单元 知识网络; 二轮专题复习的任务是, 打破章节限制, 从专题角度对知识进行梳理和串联, 对模型进行归 纳总结,训练上重在题型专项训练和大题规范训练; 三轮综合复习重在综合训练、回归基础和查缺补 漏。如何使二轮复习更好地 “承接”一轮复习并 “奠基” 三轮复习,笔者结合多年指导学生进行二轮 复习的经历, 认真总结了对二轮复习颇为有效的若千做法, 形成了此文, 希望能对高考复习备考中的同 学们有所帮助。
\end{abstract}

[关键词] 高一高二; 高考物理; 专题复习; 二轮复习; 研究

中图分类号：G633.7 文献标识码：A

\section{1 专题织网一一核心内容网 格化 \\ 二轮复习中的知识复习绝不是对一 轮复习知识的简单重复, 而是应该跳出 单元的界限, 从专题的角度总结知识, 建 议同学们画思维导图。}

\section{2 正误辨析一一基础知识习} 题化

一轮复习重在对基础知识和基本技 能进行复习。二轮复习阶段, 如果同学们 还大量重复刷题, 不但费时费力而且收 效不大, 可是基础知识确实还需要在二 轮阶段进行继续强化。为了即节省时间 又练出新意, 笔者采用了对基础知识进 行正误辨析的方式让学生训练。这些判 断正误的习题资源同学们可以在日常的 训练题当中整理出来。现以物理学史部 分为例具体说明 (表1, 限于篇幅, 部分呈 现）。

完成表格后, 为了巩固强化学生对 知识的掌握, 笔者整理了部分判断正误 题对学生进行专项训练, 现呈现部分判 断正误题供参考。

表 1 物理学史部分

\begin{tabular}{|l|l|}
\hline 物理学家 & 主要观点或贡献 \\
\hline $\begin{array}{l}\text { 亚里 士多 } \\
\text { 德 }\end{array}$ & (1) 运动需要力来维持 (2) 重的物体下落快,轻的物体下落慢 \\
\hline 伽利略 & $\begin{array}{l}\text { (1) 运动不需要力来维持 (2) 轻重物体下落一样快 (3) 实验和逻辑推理 } \\
\text { 结合的科学思维方法 (4) 发明望远镜且观察到木星的卫星 }\end{array}$ \\
\hline 笛卡尔 & 物体不受力将做匀速直线运动 \\
\hline 牛顿 & (1) 牛顿三定律 (2) 万有引力定律 (3) 相互作用抽象为力 (4) 光色散 (5) 微积分 \\
\hline
\end{tabular}

例: 正误辨析

(X) 01. 在万有引力定律的发现历 程中, 牛顿继承了开普勒和卡文迪许的 研究成果。

( $\sqrt{ }$ ) 02. 力的概念是牛顿最先提出 的。

（X）03. 牛顿根据理想斜面实验, 提 出力不是维持物体运动的原因。

( V $) 04$. 伽利略利用斜面实验延长 了小球的运动时间使得运动时间的测量 成为可能。

3 总结梳理一一经典模型明 晰化

高中物理研究的问题主要为两类, 一类直接就是模型问题, 另一类是可以 抽象为模型的物理问题, 也就是说, 高中
物理研究的问题基本都是模型问题。二 轮阶段, 同学们要学会对模型进行必要 的梳理, 抓住模型的根本, 了解其规律、 使用条件及注意事项, 配以体现模型思 想的典型习题, 这样能让同学们对模型 的掌握由浅入深, 一通百通。笔者对力学 部分精选了如 (表2) 常见模型 (限于篇幅, 部分呈现)： 彻化

\section{4 归纳领悟一一二级结论透}

高考中, 时间很关键, 要求同学们必 须在规定时间完成规定的题量, 所以高 考即考查同学们会不会, 还考查同学们 做题的速度。如何节约考试中的时间, 使得做题又快又准, 这要求同学们对知 识要非常熟悉, 熟能生巧, 除此之外, 还 
要求我们同学们要尽可能让解题模块 化。这里所说的模块实际就是 “二级结 论”, 解题中途, 只要满足相关条件, 就可 以直接得出结果, 略去了中间繁琐的思 维和数学运算, 大大节约了答题时间。这 些“二级结论”一般不会在教材中出现, 而是来源于教师的教学总结和同学们的 解题实践。二轮阶段, 同学们有必要把这 些日常总结的结论系统地翻出来细细咀 嚼, 弄清他们的使用条件, 记住他们的重 要结论, 防止用错用偏。

\section{5 积累整理一一解题方法系} 列化

物理学科对同学们的思维能力要求 比较高, 有很多有效的思维方法需要我 们同学消化理解, 笔者在教学中总结的 高中物理思维方法有：对称法, 作图法, 排除法, 等值法, 特值法, 代入法, 极限法, 量纲法, 假设法, 反证法, 比例法, 等效法, 整体法, 隔离法, 微元法等等。二轮复习 阶段, 同学们非常有必要对这些方法进 行整理, 并用经典例题对其进行认真具 体解读, 以便更好地认识和掌握这些方 法, 提升自己的解题能力。如:

思维方法示例之一一等值法:

例: 地球的同步卫星质量为 $\mathrm{m}$, 离地 面的高度为 $h$. 若地球的半径为 $\mathrm{R}$, 地球表 面处的重力加速度为 $\mathrm{g}$, 地球自转的角速 度为 $\omega$, 则同步卫星所受地球对它的万 有引力的大小为 $(\quad)$

$$
\text { A. } m(R+h) \omega^{2} \quad \text { B. } \frac{m R^{2} g}{(R+h)^{2}}
$$

\section{C. $m \sqrt[3]{\mathrm{R}^{2} \mathrm{~g} \omega^{4}} \quad$ D. 以上结果都不正确}

\section{答案: $A B C$}

感悟: $A 、 B$ 选项易推出, 对于 $C$ 选项可 由A项 $=B$ 项, 即 $m(R+h) \omega^{2}=\frac{m R^{2} g}{(R+h)^{2}}$, 求出 $(\mathrm{R}+\mathrm{h})$ 再代回 $\mathrm{A}$ 项或者 $\mathrm{B}$ 项, 但更简洁 的方法是直接利用 $A$ 项和 $B$ 项相等, 然后 利用数学规律直接相消, 即:

$$
\sqrt[3]{\left(m(R+h) \omega^{2}\right)^{2} \times \frac{m R^{2} g}{(R+h)^{2}}}=\sqrt[3]{m^{3} \omega^{4} R^{2} g}
$$

\begin{tabular}{|c|c|}
\hline 经典模型 & 模型重要说明及注意事项 \\
\hline 轻绳、轻杆 & $\begin{array}{l}\text { 1. 轻绳只提供拉力, 轻杆可以提供拉力和压力。 } \\
\text { 2. 轻绳的力一定沿绳, 轻杆的力不一定沿杆。 } \\
\text { 3. 轻杆若两点受力且平衡, 则受力沿杆。 } \\
\text { 4. 绳杆相连的物体, 沿绳杆方向速度相等。 } \\
\text { 5. 轻绳, 轻杆的力可以发生突变。 }\end{array}$ \\
\hline 轻弹簧 & $\begin{array}{l}\text { 1. 弹簧弹力满足胡克定律。 } \\
\text { 2. 弹簧弹力不可以发生突变。 } \\
\text { 3. 拉伸和压缩形变量相同时弹力大小和弹簧弹性势能相同。 }\end{array}$ \\
\hline 挂件 & $\begin{array}{l}\text { 1. 同一段绳不会出现两种力。 } \\
\text { 2. 若绳上打死结则结两边绳拉力不同。 } \\
\text { 3. 此类问题一般采用平衡分析, 用正交分解法和三角形法去解决。 }\end{array}$ \\
\hline 追击 & $\begin{array}{l}\text { 1.复杂问题应该画运动示意图。 } \\
\text { 2.可采用解析法,物理法, 相对法, 图象法分析求解。 }\end{array}$ \\
\hline $\begin{array}{l}\text { (类) } \\
\text { 坚直上抛 }\end{array}$ & $\begin{array}{l}\text { 1. 整个过程可看成是完整的匀变速直线运动,用运动学公式进行分析。 } \\
\text { 2. 可分上升阶段和下降阶段分析。 } \\
\text { 3. 上升和下降阶段具有对称性。 } \\
\text { 4. 上升阶段可采用逆向分析看成是从静止开始的自由落体。 } \\
\text { 5. 简单问题可以用匀变速直线运动的比例规律解题。 }\end{array}$ \\
\hline 板块 & $\begin{array}{l}\text { 1. 解题方法: 动力学或功能关系。 } \\
\text { 2. 关注板块的受力情况, 尤其是对摩擦力的分析。 } \\
\text { 3. 重视对物体运动状态进行分析, 关注过程中加速度是否改变。 }\end{array}$ \\
\hline (类) 平抛 & $\begin{array}{l}\text { 1. 匀变速曲线运动, 相同时间速度变化相等。 } \\
\text { 2. 可分解为两个相互垂直方向的匀速直线运动和匀变速直线运动。 } \\
\text { 3. 解题需要,还可按要求分解为两个相互垂直方向的匀变速直线运动。 } \\
\text { 4. 等时性,独立性, 等效性。 } \\
\text { 5. 运动轨迹是抛物线。 }\end{array}$ \\
\hline
\end{tabular}

这种由 A 项和B项相等推出C 项的方法即 为等值法。后面这种直接相消的方法能 大大节省解题时间, 同学们应该理解掌 握并会恰当使用。

\section{6 限时规范一一大题训练细} 节化

要想在高考中拿到高分, 大题尽可 能拿分很关键, 二轮复习阶段, 同学们要 练习快速审题和规范书写。审题要重点 关注隐含条件, 要找出关键字句, 要快速 发现解题的突破口; 做题时要坚持把题 彻底算到底, 得出最终的结果。要养成规 范书写大题的习惯, 写完大题步骤可以 让老师和同学帮你查找问题。大题规范 的书写包括, 必要的文字说明、原始公 式, 和最终结果, 文字说明一般包括交 待研究对象, 假设了什么物理量, 物理 过程是怎样的, 过程利用了什么物理规 律等等; 要用常用的符号表示物理量; 要写不带数字的原始公式; 运算结果分
数要化成小数; 如需要作图建议开始用 铅笔, 最终确认无误后再用黑色的水性 笔描一下; 画光路图要带箭头; 书写要 用黑色的水性笔以保证网上阅卷扫描 后的清晰等等。

综上所述, 二轮复习要以考试大纲 考点为中心, 以专题专项梳理为内容, 以 学生限时规范练习为手段。要做到, 凸显 学生主体, 突出一个 “练” 字; 继续关注 基础, 落实一个 “基” 字; 梳理模型方法, 感受一个 “梳” 字; 培养规范习惯, 养成 一个 “规” 字。

\section{[参考文献]}

[1] 林海雅.高考生物首轮复习 策略初探——查漏、补缺[J].中学生物 学,2012,28(06):8-9.

[2]张为禄. 浅谈中考物理总复习的策 略及方法[J].课程教育研究,2017,(37):173.

[3]许文龙.浅谈良好物理思维习惯的 培养[J].物理教学,2016,38(09):11-12. 